\title{
EL DESARROLLO DIALÓGICO DE LA ARGUMENTACIÓN MORAL Y SUS RELACIONES CON LA ÉTICA DEL DISCURSO
}

\author{
Dialogical Development of Moral Argumentation and its Relationships with \\ Discourse Ethics \\ Javier Romero \\ Universidad de Salamanca \\ jromero@usal.es
}

A Mara Manzano en su septuagésimo cumpleaños

\begin{abstract}
Resumen:
Retornando al modelo dialógico del discurso racional en la lógica dialógica y en la teoría comunicativa de Habermas, describimos la lógica dinámica como el estudio del flujo general de la comunicación. Mostramos cómo estas dos perspectivas estarían relacionadas con la teoría de los actos de habla y sus relaciones con la teoría moral. Esperamos que el presente trabajo contribuya a una mejor comprensión de la argumentación moral y sus relaciones con la ética del discurso.
\end{abstract}

\section{Palabras clave:}

Dialógica, lógica dinámica, argumentación moral, ética del discurso, Habermas

\begin{abstract}
:
Going back to the dialogical model of rational discourse in dialogical logic and Habermas's theory of communication, we describe dynamic logic as a study of general communication. We show how these two perspectives would be related to theory of speech acts and their relationships with moral theory. We hope that the following paper will contribute to a better understanding of the moral argumentation and its relationships with discourse ethics.
\end{abstract}

\section{Keywords:}

Dialogic, Dynamic Logic, Moral Argumentation, Discourse Ethics, Habermas 


\section{INTRODUCCIÓN}

Uno de los rasgos distintivos del Homo sapiens respecto de sus antepasados antropoides sería el uso del lenguaje entendido como comunicación lingüística o verbal. Si bien es cierto que los estudios en comunicación ecológica y biosemiótica distinguen acertadamente entre comunicación lingüística y comunicación no lingüistica (Dryzek, 1995, 2000; Romero, 2020), solo aquella estaría presente en los seres humanos tras un largo proceso de hominización y humanización como tempranamente Jürgen Habermas señaló en la década de los años setenta del siglo $\mathrm{XX}$, estableciendo así una diferencia entre homo faber (técnica) y homo loquens (lenguaje), que llevaría al autor a estructurar su teoría antropológica en torno a un naturalismo débil entre Kant y Darwin (Habermas, 1983b, 2008, 2011b; Romero y Mejía Fernández, 2019).

El uso de argumentos en un discurso sería una de las funciones lingüísticas que el ser humano podría realizar. A diferencia de los que consideran que la creación de discursos únicamente tendría una vertiente narrativa (como en $\mathrm{M}$. Foucault), autores como J. Habermas, K. O. Apel y la Escuela de Erlangen tendrían "como punto de partida el faktum rationis de la Argumentación" como señala Adela Cortina (Cortina, 2010: 84)'.

La acción o el proceso de contar algo sucedido realmente o una historia ficticia mediante la palabra, la escritura o medios audiovisuales y digitales, esto es, la narración, no tendría como pretensión la necesidad de demostrar ni justificar nada (la corrección o incorrección lógica no tendría sentido). En cambio, un argumento sería un segmento lingüístico que presenta cierta complejidad en el desarrollo de un razonamiento con la finalidad, mediante estructuras lógicas, de demostrar o justificar algo (premisas-conclusión). En este caso el empleo de argumentos permitiría que la previa aceptación de un enunciado se analizase en un terreno común que posibilitaría, únicamente por la argumentación y la reflexión, conducir a nuevos enunciados más coherentes y mejor construidos para su posible aceptación final (posibilidad de presentar argumentos y hacer frente a argumentos ajenos).

Desde esta perspectiva, aquellos que defienden la argumentación en la praxis comunicativa cotidiana parten de un supuesto donde los diferentes sujetos necesitan orientarse en el campo de las pretensiones de validez, incluso de las pretensiones aseverativas y normativas de validez, si quieren entenderse sobre algo dado en el mundo (Lorenzen, 1969; Apel, 1973; Habermas, 1981, 1983a). En este proceso los diferentes argumentos deben admitirse como formas de reflexión de una determinada acción orientada al entendimiento dialógico (universalismo), en la medida en que se consideran interacciones reguladas (normatividad). Es en este punto donde la noción

\footnotetext{
1 M. Foucault presenta en El orden del discurso (1970) una hipótesis de trabajo donde con influencias del estructuralismo francés, el psicoanálisis y la filosofía de Nietzsche afirma "que la producción de discurso (en toda sociedad) está a la vez controlada, seleccionada y redistribuida por cierto número de procedimientos que tienen por función conjurar sus poderes y peligros, dominar el acontecimiento aleatorio y esquivar su pesada y temible materialidad" (Foucault, 2014: 14).
} 
de significado (o regla) como uso del segundo Wittgenstein estaría muy presente (Wittgenstein, 1953).

Puesto que los distintos problemas de ética y de política acaparan hoy día buena parte del diálogo intercultural de una sociedad plural tanto en valores culturales como en convicciones morales (Gómez-Heras, 2003: 40), facilitar mecanismos de razonamiento y entendimiento intersubjetivo entre los seres humanos conduciría hacia una teoría moral donde su objetivo no sería aumentar el número de prescripciones morales, sino más bien proporcionar procedimientos lógicos que, mediante la argumentación y la aceptación de los argumentos, puedan llegar a traducir un contenido lingüístico a una estructura normativa aplicable tanto a la filosofía moral como a la teoría de la democracia; una teoría que tras el giro deliberativo de los años noventa del siglo XX se presenta como una democracia discursivo-deliberativa (Dryzek, 2000: Introducción).

Con ello volvemos a una cuestión fundamental de filosofía práctica con ecos kantianos para una sociedad pluralista y post-metafísica, basada en la pregunta: ¿por qué aceptamos las normas morales? Partiendo de esta pregunta, el siguiente trabajo de investigación tiene como uno de sus objetivos fundamentales realizar una presentación del desarrollo dialógico de la argumentación moral (de la ética monológica kantiana a la ética dialógica), es decir, analizar cómo la legitimidad de las normas morales se fundamentarían en la racionalidad de un consenso racional dialógico con carácter universal, basándose en los modelos teóricos de la lógica dialógica (de la Escuela de Erlangen a la Escuela de Lille) y sus relaciones con la teoría discursiva de la moral de Jürgen Habermas. Para ello, primeramente, se realizará una presentación histórico-filosófica de lo que Johan van Benthem ha denominado giro dinámico en la lógica actual y sus implicaciones teóricas (1), para poder realizar un análisis más adecuado del marco dialógico estándar inaugurado por la Escuela de Erlangen y radicalizado por la Escuela de Lille mediante un enfoque pragmatista de la lógica (2). Esta propuesta permitirá vislumbrar que en el marco normativo del significado de la dialógica hay presupuestos no sólo de las normas metódicas de la praxis locutoria que en la práctica posibilitaría un consenso racional (cualidades formales inherentes a la comunicación), sino además una situación hipotética de raíces contrafácticas basada en exigencias generales de simetría (reglas de partículas o reglas para jugadores anónimos) para las condiciones de equidistribución de las oportunidades de elegir y ejecutar actos de habla. Estos nuevos desarrollos en dialógica parecen tener relaciones con la ética discursiva de raíz habermasiana cuando se aceptan que los presupuestos de justificación de las normas morales no están desconectados de una manera abstracta de los supuestos ontogenéticos del proceso de hominización y humanización del Homo sapiens (3).

\section{EL GIRO DINÁMICO EN LA LÓGICA ACTUAL}

Hablar de lógica hoy implica en la mayoría de sus casos imaginar inferencias ineludibles que obligan a todos a aceptar sus conclusiones (pensemos en este caso tanto en el clásico modus tollens estoico así como en el modo de silogismo aristotélico). Desde esta perspectiva surge la pregunta de, ¿qué es la lógica? Según Hintikka y Sandu, aunque "no está claro lo que se entiende o debería entender por 
lógica, lo que sí resulta relevante, sin embargo, es identificar la lógica con el estudio de las inferencias y relaciones inferenciales", concluyendo los autores que, "en cualquier caso, tiene un evidente uso práctico: nos ayuda a razonar bien, a construir buenas inferencias" (Hintikka y Sandu, 2006) ${ }^{2}$.

Si bien los orígenes de la lógica en la antigüedad no llegan a comprenderse bien, el razonamiento sobre el debate legal, político o filosófico parece un factor clave en su aparición en las grandes tradiciones de Grecia, India, Persia y China (Rahman, Street y Tahiri, 2008: 14; van Benthem, 2014: 2). Pero los orígenes de la lógica son más variados que la concepción estática centrada en las demostraciones y en la búsqueda de la certeza absoluta mediante una metodología formal que, en el abanico de autores como Frege, Russell, el primer Wittgenstein, Ayer, Carnap o Bergman, se centró en intento de construir un "lenguaje ideal" (la filosofía como análisis del objeto de la forma lógica). Así entonces, otra imagen de la antigüedad viene dada por el debate y la polémica en la pólis griega, donde la forma que toman los diálogos platónicos nos muestra a un Sócrates sagaz frente a sus interlocutores sirviéndose de los argumentos mejor perfilados (el conocido como método socrático). Hoy el cuadro dialéctico socrático sería uno de los muchos modos de obtener información reactualizado en autores que, frente a los partidarios del "lenguaje ideal", entienden la filosofía también como análisis de la "gramática ordinaria" o uso del lenguaje. Y aquí Wittgenstein (1953), Austin (1962) y después Searle (1969), cobran importancia respecto a la teoría de los actos de habla y a lo que hoy se considera como concepción dinámica.

La combinación de lógica epistémica y dinámica produce sistemas lógicos que, en palabras de Johan van Benthem, "son capaces de describir los efectos de las acciones comunicativas mediante enunciados mixtos" (van Benthem, 2006). Un ejemplo sería: "[!A] Ki $\Phi^{\prime}$, esto es, tras anunciarse públicamente que $A$, el agente i sabe que $\Phi$. Hoy se conocen sistemas axiomáticos que son correctos y completos (estos axiomas analizan los efectos de anunciar públicamente que $\mathrm{A}$ en función de otros más simples), así como el desarrollo y la complejidad de diferentes lenguajes dinámico-epistémicos en numerosos campos de la lógica actual (van Benthem, 2006a, 2011, 2014; Rahman y Keiff, 2005). En general, más allá de la actualización de información como se daría desde una perspectiva semántica, otros procesos cognitivos jugarían un papel importante en la comunicación. Pensemos por ejemplo en el modelo más sencillo que podemos encontrar de un episodio informativo entre varios agentes, donde la pregunta y su posterior respuesta presentan características lógicas. Observemos el siguiente acto de habla:

$$
P-i \text { Esta canción es de Jimi Hendrix? }
$$

\footnotetext{
${ }^{2}$ No debemos olvidar que aplicar técnicas de deducción formal a partir de finales del siglo XIX a la lógica -un proyecto que se remonta a Leibniz-, dividirá la lógica entre formalistas (desde Boole y Frege en adelante) y no formalistas (Hegel y los que estiman que un tal cálculo y análisis carecían de valor para la filosofía). Esta división se hizo aún más fuerte a principios del siglo XX tras la polémica entre Moore/Russell frente a Bradley/McTaggart. Sobre este punto ver la obra de J. Mosterín (Mosterín, 2000: 145 y ss.).
} 


$$
\text { R - Sí, lo es. }
$$

Para van Benthem, este proceso lógico de pregunta $(\mathbf{P})$ y respuesta $(\mathbf{R})$ se repite en multitud de casos durante nuestras vidas cotidianas (van Benthem, 2006a). Aquí el agente $\mathbf{P}$ que pregunta informa que no está completamente seguro de si está escuchando una canción de Jimi Hendrix o no, al mismo tiempo que, al dirigirse a $\mathbf{R}$, queda implícito que piensa o supone que $\mathbf{R}$ puede llegar a saber si la canción es de Jimi Hendrix (transmitimos información tanto de hechos del mundo, así como de lo que sabemos acerca de otras personas). Una vez facilitada la respuesta, $\mathbf{R}$ no se limita a informar solamente de que se trata de una canción de Jimi Hendrix. Ahora sabe que $\mathbf{P}$ lo sabe, y $\mathbf{P}$ sabe eso, y así en sucesión (a partir de aquí pueden surgir otros actos de habla en la interacción). El término acuñado por filósofos, lingüísticas y estudiosos de la teoría de juegos para estos casos sería: $\mathbf{P}$ y $\mathbf{R}$ han alcanzado conocimiento compartido del hecho Jimi Hendrix. Si este ejemplo puede parecer menor, recurramos al que van Benthem ha presentado respecto a imaginar que $\mathrm{x}$ ha descubierto el número secreto de la cuenta bancaria de $y$. Si $x$ sabe que $y$ no sabe que $x$ conoce el número, $x$ caerá en la tentación de entrar en la cuenta bancaria de $y$; pero si por el contrario $x$ sabe que y sabe que $x$ conoce el número, probablemente $x$ se abstenga de entrar en la cuenta bancaria de $y$. "nuestro comportamiento lo gobierna la información que compartimos" (van Benthem, 2006a).

Estos ejemplos serían válidos para la Pragmática Universal de Jürgen Habermas (Habermas, 1981, 2011a, 2011b). Para el autor, la lógica de la argumentación no se refiere a relaciones de inferencia entre unidades semánticas (semantischen Einheiten), sino a relaciones de inferencia entre unidades pragmáticas (pragmatischen Einheiten) representadas en los actos de habla presentes en todo proceso de argumentación en la forma de actitudes ilocucionarias que tendrían un carácter imperativo, constatativo, regulativo, expresivo y comunicativo, según su propio desarrollo dinámico en la interacción (Habermas, 1981: 45 y ss., 433 y ss.) ${ }^{3}$. En un artículo donde alude a la teoría comunicativa de Habermas, van Benthem se hace la siguiente pregunta: where is logic going, and should it? (¿a dónde va la lógica, y debería?) (van Benthem, 2006b). En concreto el autor señala que, en la fragmentación de la filosofía moderna en numerosas filosofías, la lógica habría sucumbido por igual a este proceso (aunque más tarde) en lo que se conoce hoy como "pluralismo lógico". A este análisis señalamos que las críticas del segundo Wittgenstein y Austin a la concepción estática de la lógica, así como las insuficiencias del realismo clásico (desde Aristóteles al último Russell), sin olvidar en este caso el teorema de incompletud de la aritmética forma/ de Gödel, socavaron por igual algunas creencias sobre el carácter inmutable (y monista) de la lógica en la búsqueda de la certeza absoluta y de una fundamentación segura para la lógica y las matemáticas.

A estas alturas queda claro que no existe dicha fundamentación. La verdadera estabilidad y el éxito de nuestras prácticas cognitivas tiene que

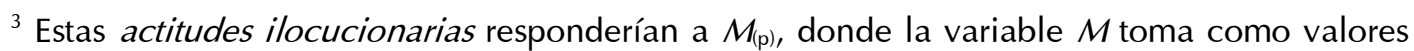
los dispositivos indicados de fuerza ilocucionaria y (p) expresiones que representan el componente proposicional.
} 
ver con la manera interactiva y dinámica con la que procesamos la información, y con la capacidad de nuestros mecanismos adaptativos para conseguir aquellas creencias que se vuelven problemáticas (van Benthem, 2006b).

Para van Benthem esto supone hablar de un giro dinámico en la lógica moderna que, en este caso, adoptaría la forma de un triángulo (van Benthem, 2006a, 2006b, 2011 , 2014). En los distintos vértices del triángulo se encontraría la teoría lógica (el lenguaje), la realidad empírica de las prácticas habituales de razonamiento y actualización de información (ya sea en semántica o en pragmática), así como también un terreno de diseño de nuevas prácticas, con frecuencia virtuales, inspiradas por la teoría de la computación y los desarrollos desde Turing (Figura 1).

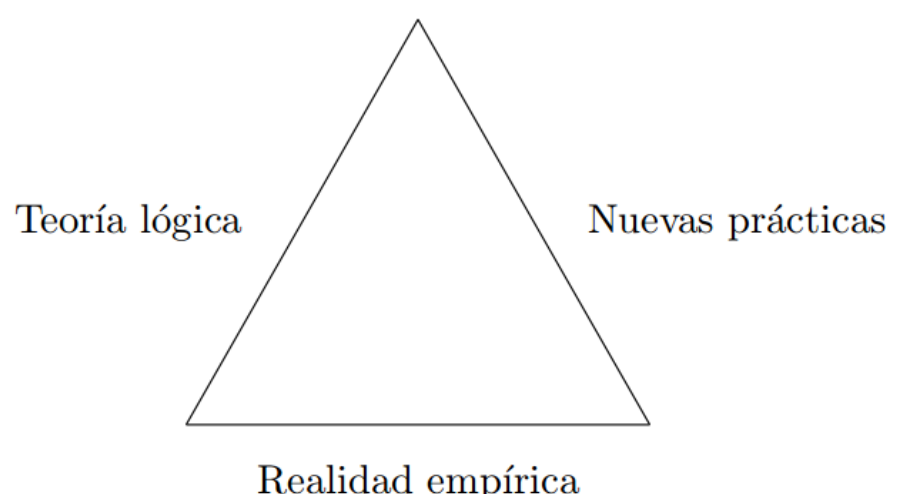

Figura 1: Triángulo dinámico-epistémico en J. van Benthem

Este proceso de democratización de la lógica (diferentes lógicas, nuevas prácticas y realidad empírica), conduciría a la siguiente pregunta: ¿es posible otra semántica?, es decir, ¿otra forma de entender el significado? Desde el segundo Wittgenstein sabemos que, en muchos casos, comprender el significado de una expresión significa conocer el uso que se hace de esa expresión en un determinado contexto de interacción lingüística, que a su vez es entendido como un juego (Wittgenstein, 1953). Aunque en este caso Wittgenstein nunca ofreció una definición precisa de juego del lenguaje puesto que entendía que estos juegos en la mayoría de sus casos carecían de reglas y, por lo tanto, de forma, la lógica clásica presenta una fuerte ambivalencia como señala Peter Martin-Löf: "un mismo término designa a la vez una acción y el contenido o resultado de dicha acción" (Martin-Löf, 1996). Acciones cognitivas como el juicio, que representaría tanto una acción intelectual como el contenido de esa acción, así como el razonamiento, que denota tanto un proceso intelectual como sus distintas aplicaciones, e incluso nuestro uso ordinario del término "lenguaje natural", tienen todos ellos un carácter dual. Esta interacción entre contenidos estáticos y acciones dinámicas estaría presente no sólo en las estructuras lógicas estáticas de proposiciones, palabras y reglas, sino también en la actividad social dinámica representada en muchas convenciones que no estarían necesariamente codificadas en una sintaxis específica (van Benthem, 1991). 
Esa dualidad entre el contenido estático, por una parte, y la acción dinámica, por otra parte, no hace sino confirmar que la estructura proposicional de la tradición clásica (estática) estaría definida semánticamente como una estructura booleana donde las proposiciones son consideradas como valores de verdad y las constantes lógicas como operadores sobre esos valores; algo que algunos autores ven problemático para una semántica de raíces intuicionistas después de la crítica a la doble negación y al tercero excluido (Redmond y López-Orellana, 2018).

Si bien para la estructura proposicional de la lógica clásica se proporciona una semántica sólida desde los resultados obtenidos por A. Tarski, cuya formulación conduciría a la teoría lógica altamente abstracta denominada Teoría de Modelos ${ }^{4}$, desde esta perspectiva dinámica lo problemático de la definición tarskiana de modelo presupone la validez del tercer excluido, "y por lo tanto la lógica intuicionista emerge como un cálculo puro sin que se le pueda asociar a una semántica entendida en el sentido de una teoría de la referencia" (Redmond y LópezOrellana, 2018). Esta perspectiva coincide con la crítica de Habermas a la lógica y a la filosofía del lenguaje que, desde Carnap, se orientó exclusivamente a las nociones sintácticas y semánticas de los productos simbólicos, sin prestar atención a una pragmática del lenguaje que habría sido desplazada de la filosofía favoreciendo únicamente los análisis del lenguaje en términos sintácticos, semánticos y fonéticos: la "falacia abstractiva" como la denomina el propio Habermas.

Pero este corte analítico no debe llevar a la idea de que la dimensión pragmática del lenguaje, de la que se hace abstracción, no puede ser sometida a un análisis formal. Considero que se está cayendo en una falacia abstractiva cuando el éxito o, al menos, las importantes perspectivas hoy abiertas en la reconstrucción de sistemas de reglas lingüísticas, se consideran como una circunstancia que justifica limitar el análisis formal a ese ámbito objetual (Habermas, 2011a: 303-304).

En efecto, el empleo de oraciones y emisiones sería accesible a un análisis formal. Separar los niveles analíticos de "lengua" y "habla" sería un error (abstracción

\footnotetext{
${ }^{4}$ La teoría semántica de Tarski estaría estrechamente relacionada con la Teoría de Modelos, es decir, la rama lógico-filosófica que se ocuparía de las relaciones entre las estructuras matemáticas y los lenguajes formales. El esquema de la Teoría de Modelos sería el siguiente: tenemos un lenguaje $L$ y una clase de objetos $M$ que son los sistemas, y entre estos dos tipos de realidades tendemos un puente: la noción de "verdad" (thruth in a structure). Gracias a Gödel, Tarski, Henkin, Robinson, Vaught, Craig, Löwenheim-Skolem y Addison, muchos de ellos miembros del Group in Logic and Methodology of Science de la Universidad de Berkeley, California, las contribuciones filosóficas a este campo fueron relevantes para la epistemología en términos semánticos (Addison, Henkin y Tarski, 1965; Hodges, 1986; Manzano, 1989). Habermas valora la noción de "verdad" de Tarski para explicar los métodos habituales de investigación y elección de teorías científicas como señala en Verdad y justificación (1999) (Habermas, 2011: 242-244). Por otro lado, desde el estructuralismo metacientífico se han logrado sólidos resultados para el análisis sincrónico de teorías científicas sirviéndose de la Teoría de Modelos (Ulises Moulines, 1996; Díez y Ulises Moulines, 2016: 297 y ss.).
} 
idealista), que conduciría a menospreciar la dimensión pragmática del lenguaje, "abandonada a un análisis exclusivamente empírico", es decir, "a ciencias empíricas tales como la psicolingüística o la sociolingüística" (Habermas, 2011a: 304). En este punto tenemos una propuesta que culmina en la Pragmática Universal, donde no sólo las unidades elementales del lenguaje pueden analizarse en la actitud metodológica de una ciencia reconstructiva (oraciones semánticas), sino también las unidades elementales de habla (emisiones pragmáticas) (Habermas, 1981, 2011a: 299 y ss.). Aquí existiría un punto en común entre la dialógica y la teoría de Habermas basado en entender que el lenguaje no estaría exclusivamente orientado hacia una descripción de hechos (semántica), sino también hacia el diálogo (pragmática); algo que Habermas valora en el trabajo de P. Lorenzen respecto a las normas metódicas de la praxis locutoria (Habermas, 1983b: 271).

En este punto las relaciones entre la dialógica y la Pragmática Universal parecen tender un puente cuando la dialógica es entendida como un enfoque pragmatista de la lógica (el tablero donde juegan distintas lógicas). Veamos a continuación el marco dialógico estándar desde los desarrollos de la Escuela de Erlangen hasta los últimos resultados teóricos de la Escuela de Lille, para una mejor comprensión de este posible trabajo conjunto en referencia a la argumentación moral y la ética del discurso como se presentará en el último apartado.

\section{MARCO DIALÓGICO ESTÁNDAR: DE LA ESCUELA DE ERLANGEN A LA ESCUELA DE LILLE}

La dialógica se inició a finales de la década de los años cincuenta del siglo XX en el marco filosófico del programa constructivista de la Escuela de Erlangen por $P$. Lorenzen y después por K. Lorenz, inspirados ambos por la noción de significado como uso de Wittgenstein, como resultado de solucionar algunos de los problemas surgidos en la obra Operative Logik (1955) de Lorenzen (Lorenzen, 1955, 1969; Lorezen y Lorenz, 1978; Rahman y Keiff, 2005; Redmond y Fontaine, 2011; van Benthem, 2014: 363 y ss.). La idea básica de la dialógica sería demostrar que el significado de las constantes lógicas estaría dado por las normas o reglas para su uso, y que estas reglas deben entenderse como las formas específicas de la interacción argumentativa (teoría pragmática del significado). En dialógica, las reglas que fijan el significado pueden llegar a ser de más de un tipo, determinando la reconstrucción de una práctica argumentativa y/o lingüística que una cierta forma de juegos del lenguaje, Ilamado en este caso diálogos, proporciona.

La adopción de una perspectiva pragmática de la lógica a partir de la protológica de Lorenzen, radicalizaron la dialógica a principios de los años noventa del siglo XX por uno de los discípulos de Lorenz, Shahid Rahman. Rahman ha logrado en las últimas décadas sistematizar la dialógica con el fin de desarrollar y combinar diferentes enfoques lógicos, el tablero donde juegan distintas lógicas (un proyecto que históricamente ha logrado ir más allá de la propuesta clásica de Lorenzen y Lorenz). Gracias a Rahman y a su equipo de investigación en la Universidad de Lille, la idea de desarrollar un marco semántico dentro de la pragmática lingüística estaría teniendo sólidos resultados no sólo para la lógica clásica e intuicionista, sino también para las lógicas no clásicas. A continuación se presentará brevemente el marco dialógico estándar mediante una descripción formal de la dialógica dada por 
la lógica proposicional que serviría tanto para la lógica clásica como para la lógica intuicionista (Rahman y Keiff, 2005: 365 y ss.). Para un ejemplo de resolución dinámica de un diálogo condicional, ver el Anexo que acompaña al texto.

\subsection{El lenguaje de la dialógica}

Sea $L$ un lenguaje de primer orden construido en base de conectivas proposicionales $(\wedge, \vee, \rightarrow, \neg)$, cuantificadores $(\forall, \exists)$, pequeñas letras para fórmulas principales $(a, b$, $C, \ldots)$, letras en mayúscula y cursiva para fórmulas que pueden llegar a ser complejas (A, B, C,...), letras en mayúsculas, cursiva y negrita para predicados $(A, B, C, \ldots)$, que las constantes sean anotadas ( $\mathrm{i}$ ), donde $i \in \mathrm{N}$, y por variables de uso $(\mathrm{x}, \mathrm{y}, \mathrm{z}, \ldots)$, la dialógica amplía el lenguaje $\mathrm{L}$ con dos etiquetas: $\mathbf{P}$ (Proponente) y $\mathrm{O}$ (Oponente), que correspondería a los participantes en un diálogo ${ }^{5}$. Las acciones o movimientos en un diálogo implican enunciados declarativos, enunciados interrogativos, peticiones, etc., es decir, actos de habla.

En un diálogo entrarían elementos de ataque (ataques de $\mathrm{O}$ realizados por medio de fórmulas o preguntas [?]) y defensa argumentativa después de ser entrega la tesis o fórmula lógica (defensas de $\mathbf{P}$ realizadas únicamente por medio de fórmulas para defender la tesis propuesta [!]) ${ }^{6}$. La esquematización del diálogo mediante una tabla de juego (game board) es un elemento de visualización del desarrollo dialógico como se puede observar en el ejemplo presentado en el Anexo que acompaña al texto. Cuando la identidad de un jugador no importa se utilizan variables como $X$ o $Y$ para $\mathrm{P}, \mathrm{O}$, siendo $X \neq Y$ (simetría e igualdad). Al afirmar una fórmula, un jugador $X$ se compromete con su retador $Y$ a defender la fórmula contra los distintos ataques permitidos a $Y$. Una jugada es una expresión de la forma $\mathrm{Xe}$, donde e es bien una

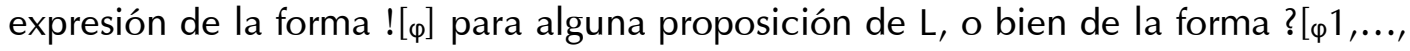
$\left.{ }_{\varphi} n\right]$. Después de presentado $L$, la dialógica presenta una serie de reglas.

\subsection{Las reglas dialógicas}

\subsubsection{Reglas de partículas (o reglas para jugadores anónimos)}

En dialógica las reglas de partículas establecen el significado local, esto es, reglas que regulan las jugadas que constituyen peticiones o requerimientos (peticiones de

\footnotetext{
${ }^{5}$ El diálogo aquí debe ser entendido como el espacio donde $\mathbf{P}$ y $\mathbf{O}$ discuten y argumentan una tesis determinada (o fórmula lógica). Este proceso estaría regido por ciertas reglas previamente fijadas como veremos más adelante (reglas de partículas o reglas para jugadores anónimos y reglas estructurales).

${ }^{6}$ Atacar o defender con fórmulas debe ser entendido en dialógica como la acción de proferir fórmulas (uttering).
} 
$\mathbf{O}$ a $\mathbf{P})$, y aquellas que son respuestas (respuestas de $\mathbf{P}$ a las peticiones de $\mathbf{O}$ ). Las reglas de partículas para operadores lógicos serían (Cuadro 1$)^{7}$ :

\begin{tabular}{|l|c|c|c|}
\hline & Afirmación $(\mathbf{P})$ & Ataque $(\mathbf{O})$ & Defensa $(\mathbf{P})$ \\
\hline Conjunción $(\wedge)$ & $\mathbf{P} ![\varphi \wedge \Psi]$ & $\mathbf{O} ?[\varphi] o \mathbf{O} ?[\Psi]$ & $\mathbf{P} ![\varphi] o \mathbf{P} ![\Psi]$ \\
\hline Disyunción $(\vee)$ & $\mathbf{P} ![\varphi \vee \Psi]$ & $\mathbf{O} ?[\varphi, \Psi]$ & $\mathbf{P} ![\varphi] o \mathbf{P} ![\Psi]$ \\
\hline Condicional $(\rightarrow)$ & $\mathbf{P} ![\varphi \rightarrow \Psi]$ & $\mathbf{O} ![\varphi]$ & $\mathbf{P} ![\Psi]$ \\
\hline Negación $(\neg)$ & $\mathbf{P} ![\neg \varphi]$ & $\mathbf{O} ![\varphi]$ & $\otimes$ \\
\hline Universal $(\forall)$ & $\mathbf{P} ! \forall x[\varphi]$ & $\mathbf{O} ?\left[\varphi\left(x / a_{i}\right)\right]$ & $\mathbf{P !}\left[\varphi\left(x / a_{i}\right)\right]$ \\
\hline Existencial $(\exists)$ & $\mathbf{P} ! \exists x[\varphi]$ & $\mathbf{O} ?\left[\varphi\left(x / a_{n}\right)\right]$ & $\mathbf{P !}\left[\varphi\left(x / a_{i}\right)\right]$ \\
\hline
\end{tabular}

Cuadro 1: Reglas de partículas o reglas para jugadores anónimos

En este punto no hay que olvidar que las reglas de partículas proporcionan una descripción abstracta sobre cómo se procede en el diálogo a nivel local: especifican el modo en el que una proposición puede atacarse [?] o defenderse [!] de acuerdo con su constante lógica. Rigurosamente, las expresiones que aparecen en el Cuadro 1 no serían jugadas reales al carecer de jugadores específicos y servirían solamente para una presentación esquemática. Estas reglas para jugadores anónimos o reglas simétricas servirían también para evitar cualquier acción estratégica en un diálogo, ayudando así a constituir el enfoque normativo del significado del marco dialógico como se verá más adelante.

\subsubsection{Reglas estructurales}

En dialógica las reg/as estructurales establecerían el significado local, esto es, reglas que determinan el curso y organización del diálogo. Serían las condiciones exactas donde una fórmula genera un juego dialógico que proporcionaría un método de decisión. Las reglas estructurales (en adelante "SR"), podrían clasificarse de la siguiente manera ${ }^{8}$ :

1) SRO (Regla de inicio) ${ }^{9}$

Cualquier partida de $p$ en $\mathrm{D}_{(\varphi)}$ comienza con $\mathrm{P} !_{(\varphi)}$. Llamamos $\varphi$ a la tesis de la partida y del juego dialógico correspondiente (tema de la argumentación). El

\footnotetext{
${ }^{7}$ En este cuadro, una expresión de tipo $a_{i}$ es una constante individual y $\varphi\left(a_{i} / x\right)$ expresaría la proposición obtenida mediante la sustitución de cada ocurrencia de $x$ en $\varphi$ por $\mathrm{a}_{i}$. Cuando

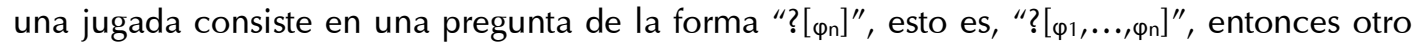
jugador elige una proposición entre $\varphi_{1}, \ldots, \varphi_{n}$ y la juega. Esto permitiría distinguir la conjunción $(\wedge)$ y disyunción $(\vee)$, por una parte, y la cuantificación universal $(\forall)$ y existencial $(\exists)$, por otra. Señalar que en el $(\exists)$ de "P! $\left[\varphi\left(x / a_{i}\right)\right]$ ", tenemos que $1 \leq i \leq n$.

${ }^{8}$ Un juego dialógico para $\varphi$, escrito $D_{(\varphi)}$, sería el conjunto de todas las partidas con $\varphi$ como tesis o fórmula principal.

${ }^{9} \mathrm{Si}$ la tesis no es una proposición compleja, sino que es una proposición elemental, entonces la regla estructura/ que hay que aplicar no es la SR0 sino la SR2.
} 
diálogo comienza con la tesis o fórmula principal, y cada movimiento que sigue será un ataque [?] o una defensa [!].

2) SR1 (Regla clásica)

La primera parte de esta regla establece que cada movimiento, después de la elección de los rangos de repetición es bien un ataque [?] o bien una defensa [!].

$$
\begin{gathered}
\text { Sea } P \in D_{(\varphi)} \text {. Para cada } M \text { en } p \text { donde } p_{p}(M)>2 \text { tenemos } F_{p}(M)=\left[m^{\prime}, Z\right] \\
\text { donde } m^{\prime}<p_{p}(M) y Z \in[a, d] \text {. }
\end{gathered}
$$

La segunda parte asegura la finitud de las partidas mediante el establecimiento de un rango de repetición del jugador como el número máximo de veces que puede desafiar o defenderse de un movimiento determinado de otro jugador.

Sea r el rango de repetición del jugador $X$ y $p \in D_{\varphi}$ ) tal que el último miembro de $p$ es una jugada de $Y, M_{0}$ es una jugada de $Y$ de posición $m_{0}$ en $\pi, M_{1}, \ldots, M_{n}$ son las jugadas de $X$ en $p$ tal que $F_{p}\left(M_{1}\right)=\ldots=F_{p}\left(M_{n}\right)=\left[m_{0}, Z\right]$.

\section{3) SR1i (Regla intuicionista)}

Los jugadores solo pueden defenderse del último de los ataques aún no respondidos, es decir, en cualquier movimiento a cada jugador le es permitido atacar una formula. La SR1i impone un modo de juego más restrictivo que el de la lógica clásica respecto a la elección de una u otra jugada durante el desarrollo de una partida.

4) SR2 (Regla formal)

Esta regla es una de las más sobresalientes de la dialógica: si el proponente (P) afirmó una proposición elemental, entonces el oponente $(\mathbf{O})$ la afirmó ya antes. Se remonta a la reconstrucción de la dialéctica platónica llevada a cabo por Aristóteles. La idea principal es que, cuando una proposición elemental es atacada [!], entonces desde el punto de vista puramente argumentativo la única respuesta posible es apelar a las concesiones de $\mathbf{O}$ (jugadas espejo).

Sea $\varphi$ una proposición elemental, $N$ la jugada $P ![\varphi]$ y $M$ la jugada $O ![\varphi]$. Una secuencia $p$ de jugadas es una partida sólo si se cumple: si $N \in p$ entonces $M$

$$
\in p \text { y } p_{p}(\mathrm{M})<p_{p}(\mathrm{~N}) \text {. }
$$

5) SR2* (Regla formal modificada) 
O puede atacar una proposición atómica si, y sólo si, él mismo aún no la afirmó. P se defiende de un ataque mostrando que, en el desarrollo del juego, O será forzado a conceder la proposición atómica atacada, digamos en el movimiento $n$. En cuanto $\mathrm{O}$ jugó $n$, entonces $\mathbf{P}$ se defiende del ataque, respondiendo sic (n); esto puede leerse como: porque tú mismo acabas de conceder en n la proposición atómica buscada ${ }^{10}$.

6) SR3 (Regla de ganancia)

Decimos que una partida está terminada o finalizada cuando no puede ampliarse en sucesivos movimientos legales, es decir, decimos que $X$ termina cuando el último movimiento en la partida es un movimiento del jugador $X$.

X gana sí, y sólo si, es el turno de Y pero no tiene ningún movimiento posible -ya sea de ataque [?] o de defensa [!]-.

Tras la resolución argumentativa $\mathbf{P}$ y $\mathbf{O}$ realizan el movimiento, esto es, la oración realizativa en el sentido clásico de Austin u "oración performativa" (performative sentence) (Austin, 1962: 6). Un ejemplo práctico siguiendo este marco estándar en el Anexo que acompaña al texto.

\section{ARGUMENTACIÓN MORAL Y ÉTICA DEL DISCURSO}

Visto lo anterior, entendemos que los diálogos son juegos de lenguaje formalmente definidos "para que establezcan la interfaz entre la actividad lingüística concreta y la noción formal de demostración" (Redmond y López-Orellana, 2016). Proponente (P) y oponente $(\mathbf{O})$ intercambian movimientos que son concretamente actos lingüísticos donde la praxis cotidiana engloba distintos tipos de acción guiada por reglas, entendiendo que los discursos y los actos de habla se distinguen unos de otros según el lenguaje utilizado: no es lo mismo un acto de habla constatativo sobre la exposición de estados de cosas con una actitud objetiva y una pretensión de validez orientada a la "verdad" en el mundo objetivo, que un acto de habla regulativo sobre el establecimiento de relaciones interpersonales con una actitud de conformidad a las normas y una pretensión de validez orientada a la rectitud normativa en el mundo social (Habermas, 1981: 439). Por lo demás, las acciones se distinguirían en sociales y no-sociales (o instrumentales). La acción social consistiría en una interacción normativamente regulada entre agentes que operan comunicativamente, o bien en un intento de ejercer influencia mutua entre oponentes que operan estratégicamente. Por otra parte, la acción no-social, a pesar de estar inmersa en contextos de acción social, serviría para llevar a cabo intervenciones finalistas en el mundo de las cosas y en sucesos encadenados causalmente (la acción instrumental) (Habermas, 1981, 2011a, 2011b: 23 y ss.).

${ }^{10}$ Esta regla formal modificada no se usa para una dialógica estándar. 
En general, un actor que sigue wittgensteinianamente una regla (o la vulnera) debería tener una visión clara o intuitiva sobre el tipo de regla que está siguiendo o vulnerando. Como bien señala Habermas, "no hay un sentido «deontológico» unitario en la obligatoriedad normativa" (2011b: 23), a lo que entendemos que en este caso vulnerar las reglas de la lógica, de la geometría y de la aritmética, así como las reglas de medición de fenómenos físicos y reglas gramaticales que sirven todas ellas para la creación y la ordenación sintáctica de constructos simbólicos mediante signos, figuras, números, cálculos, oraciones, etc., solamente tendría consecuencias intrínsecas a su propia práctica al no estar referidas todas ellas a algo externo a la propia praxis.

Cuando erramos la «lógica» o el sentido propio de una práctica como ésta, en cierta medida fracasamos por causa de nuestra incompetencia «en» la regla misma. Pero nadie nos castiga: ni la propia conciencia, ni la sociedad, ni la naturaleza (Habermas, 2011b: 24).

Si en el sentido intrínseco ni la propia conciencia, ni la sociedad, ni incluso la naturaleza sancionan la vulneración de una regla, en un sentido extrínseco las normas de acción social tienen el carácter "deontológico" de obligar a los destinatarios a seguir la regla. Aquí la posible sanción ante una vulneración, a diferencia del sentido intrínseco, varía con el tipo de regla, ya sea en la vulneración de las normas morales o jurídicas, o infringiendo costumbres u convenciones, o en su caso desviándose de los roles sociales asignados puesto que "tales normas regulan las relaciones interpersonales de actores que se comunican entre ellos y que participan en prácticas comunes", sin olvidar que "estas prácticas constituyen elementos inmediatos del contexto simbólico de un mundo de la vida" (Habermas, 2011b: 24).

A partir de este punto la noción kantiana de "razón práctica", en una sociedad plural y post-metafísica se divide para Habermas en tres usos: uso pragmático, uso ético y uso moral (Habermas, 1983a, 2000: 109 y ss.). Puesto que el uso ético procedería monológicamente (como ocurriría en Kant), el uso moral en cambio requiere por parte de los sujetos una perspectiva intersubjetiva y dialógica, limitando su campo de aplicación práctica a las cuestiones relativas a lo justo, esto es, "defender la primacía de lo justo entendido deontológicamente sobre lo bueno" (Habermas, 2000: 9). Así entonces, la teoría moral del discurso (o ética discursiva) estaría fuertemente respaldada por la estrategia de la regla de la argumentación del "principio de Universalidad" (U) de la Pragmática Universal. Aquí una norma tendría "validez" sí, y sólo si, los participantes en un discurso práctico llegan (o pueden llegar) a un acuerdo sobre una práctica moral. Este "postulado ético discursivo" (D) ya presupone para Habermas que se pueden fundamentar normas que lleven a su cumplimiento debido al carácter inclusivo de la teoría comunicativa donde todos los afectados por una norma " $\mathrm{x}$ " deben formar parte del diálogo para su posible resolución final (Habermas, 1981, 1983a: 76 y ss.).

Si bien es verdad que algunos autores consideran la Pragmática Universal de Habermas como débil debido al apoyo teórico que el autor encuentra en las ciencias empíricas (García Marzá, 1992), no hay que olvidar que su teoría está anclada en las ciencias naturales y en la antropología biológica desde antes incluso de Teoría de la 
acción comunicativa (Habermas, 1983b; Romero y Mejía Fernández, 2019). De esta manera la regla de argumentación del "principio de universalidad" (U) de la Pragmática Universal en la que se basa la ética del discurso presupone estructuras argumentativas en el Homo sapiens de entender el significado como uso como la dialógica mantiene. En efecto, encontramos en el desarrollo teórico puro semejanzas entre Habermas y la dialógica siempre que la lógica normativa se mantenga, según Lorenzen, en un "sistema cerrado de filosofía pura" pero, y aquí estaría la novedad que los defensores de la lógica dinámica han señalado en numerosas ocasiones, "abierto para todas las aplicaciones" (Lorenzen, 1969: 89).

Ese interés por la teorización pura y la sistematización de la acción humana abarcaría ese conjunto de lógicas de la interacción donde Habermas, Apel y la dialógica convergen. Como vimos en el anterior apartado, desde Rahman la dialógica se ha orientado por completo hacia la argumentación y la práctica lingüística proporcionando ese tablero dialógico para las distintas lógicas mediante un principio de carácter normativo representado por las "reglas lógicas" (reglas de partículas o reglas para jugadores anónimos y reglas estructurales). Por todo ello, al consenso intersubjetivo se llegaría mediante la coacción sin coacciones del mejor argumento en sentido habermasiano (Habermas, 1981, 1983a, 2011b) o mediante las reglas estructurales de la Escuela de Lille: un diálogo argumentativo finaliza cuando no se pueden ampliar en sucesivos movimientos legales ( $X$ gana sí, y sólo si, es el turno de $Y$ pero no tiene ningún argumento posible) (Rahman y Keiff, 2005; Redmond y Fontaine, 2011).

En el consenso argumentativo (donde $X$ e $Y$ aceptan una tesis conjuntamente), según Habermas y la dialógica los sujetos realizarían el movimiento (o acción), esto es, la "oración performativa" que se remonta a Austin. Además, un marco dialógico enriquecido con objetos lúdicos presenta nociones previas de igualdad entre sus participantes, "que es consecuencia de un nivel más fundamental de significado en el que las reglas se formulan independientemente del jugador que las aplica" (Rahman y Redmond, 2016). Este proceso guarda una cierta similitud con esa situación hipotética que ya Habermas caracterizaba respecto a su teoría de acción comunicativa como "situación ideal de habla".

Llamo ideal a una situación de habla en que las comunicaciones no solamente no vienen impedidas por influjos externos contingentes, sino tampoco por las coacciones que se siguen de la propia estructura de la comunicación. La situación ideal de habla excluye las distorsiones sistemáticas de la comunicación. Y la estructura de la comunicación deja de generar coacciones sólo si para todos los participantes en el discurso está dada una distribución simétrica de las oportunidades de elegir y ejecutar actos de habla. De esta exigencia general de simetría pueden deducirse para las distintas clases de actos de habla exigencias especiales de equidistribución de las oportunidades de elegir y ejecutar actos de habla (Habermas, 2011a: 153).

Estos niveles de "situación ideal de habla" en sentido habermasiano o en términos dialógicos la situación previa de las "reglas de partículas" (o reglas para jugadores 
anónimos para $X$ e $Y$, donde $X \neq Y$, muestran que el desarrollo dinámico posterior (en dialógica basado en las reglas estructurales) sería el resultado de una regla local de igualdad única formulada como una norma argumentativa para jugadores anónimos en relación de simetría. Hay que destacar que, desde los desarrollos teóricos de la Escuela de Lille, la incorporación de la "regla socrática" a partir de la Teoría Constructiva de Tipos (TCT) ayudaría a constituir y reforzar el enfoque normativo del significado del marco dialógico (Rahman y Redmond, 2016) ${ }^{11}$. Estos desarrollos previos a la argumentación garantizarían la no-coacción externa mediante una distribución simétrica de elegir y ejecutar actos de habla apoyados por variables anónimas como $\mathbf{P}$ y $\mathbf{O}$. Aquí, igual que ocurriría con la "situación ideal de habla" de Habermas, intuimos principios de justicia e igualdad a la hora de elegir y ejecutar actos habla.

Estos distintos avances teóricos podrían ser aplicados a una teoría moral universalista mediante la (dia)lógica de la argumentación moral como la ética del discurso propone. Tal como señala Adela Cortina siguiendo a Lorenzen, la teoría moral no respondería inmediatamente a la pregunta monológica, ¿qué debo hacer?, sino a la pregunta dialógica, ¿por qué debo? Es en esta pregunta donde la filosofía moral muestra la racionalidad que existe en el obrar humano (acción comunicativa), al tratar "de esclarecer si es acorde a la racionalidad humana atenerse a la obligación universal expresada en los juicios" (Cortina, 2010: 77). La argumentación moral, por tanto, estaría orientada a dar razones que intenten responder a la pregunta de ipor qué debo?

Una respuesta a esta pregunta quizá vendría estructurada desde la pragmática habermasiana y la dialógica, que mediante sus metodologías ofrecerían un marco pragmático de argumentación que en su aplicación práctica sirve también de criterio para la preferencia racional de códigos morales. Aún así, Habermas ha señalado respecto a Lorezen (e incluso respecto a Rawls y Apel) que aún estando de acuerdo con las tesis de las normas metódicas de la praxis locutoria que en semejantes cuestiones prácticas posibilitan un consenso racional, "toda teoría general de justificación permanece característicamente abstracta frente a las formas históricas de la dominación legítima" (Habermas, 1983b: 271). En efecto, Habermas ha señalado que aplicar distintos raseros de justificación discursiva a sociedades tradicionales sería comportarse de un modo históricamente injusto (pensemos en las sociedades del neolítico o en las sociedades medievales, por ejemplo). ¿Hay una alternativa a esa injusticia histórica de las teorías generales, por una parte, y a la arbitrariedad de la mera comprensión histórica?, se pregunta el autor. El único programa con perspectivas viene dado para Habermas por una teoría que aclarase estructuralmente la sucesión, históricamente observable, de niveles de justificación que puedan ser

\footnotetext{
${ }^{11}$ Esta regla se remontaría a la reconstrucción que hace Aristóteles de la dialéctica platónica: cuando una proposición elemental es atacada [?] entonces -desde el punto de vista puramente argumentativo- la única respuesta posible es apelar a las condiciones del oponente (O), es decir, sin hacer uso de la autoridad más allá de los movimientos presentados durante la interacción argumentativa (Rahman y Redmond, 2016).
} 
reconstruidos en un contexto lógico-evolutivo gracias a Darwin y a los resultados empíricos de la teoría de la evolución por selección natural en distintos campos (genética, antropología, psicología, lingüística, etc.), como el propio Habermas presentó hace décadas (Habermas, 1983b, 2008, 2011b; Romero y Mejía Fernández, 2019).

Estos procesos vendrían verificados indirectamente por la psicología evolutiva de signo cognoscitivo (Piaget y Kohlberg) que reconstruye, en lo relativo a la ontogénesis del Homo sapiens, las etapas de conciencia moral en clave constructivista haciendo posible entender el desarrollo moral teleológicamente orientado hacia la asunción de unos principios éticos universales que toda la humanidad debiera seguir (Habermas, 1983a: 76 y ss.). Estos principios éticos universales vendrían dados tras un largo proceso de humanización que históricamente se podría reconstruir gracias a una teoría genética de la acción entendida desde:

a) las estructuras generales de acción (que fundamentan la situación normal, escasamente conflictiva) y las estructuras nucleares (que posibilitan la solución consensual de los conflictos), regidas ambas por la conciencia moral (Piaget y Kohlberg),

b) estructuras de las imágenes del mundo determinantes para la moral y el derecho, y

c) estructuras del derecho institucionalizado e ideas morales vinculantes.

Esta división estaría presente, para Habermas, en las sociedades del neolítico, las primeras civilizaciones, las civilizaciones desarrolladas y la modernidad, con sus respectivas diferencias (transición desde el Paleolítico hasta el Neolítico y la aparición de las primeras ciudades y Estados), en su peculiar reconstrucción del materialismo histórico más allá de K. Marx (Habermas, 1983b: 159; Anderson, 2013: 79 y ss.; Romero y Mejía Fernández, 2019). Con esta teoría antropológica en mente es posible una explicación sobre las etapas morales y la asunción de principios universales que, en las sociedades pluralistas y democráticas de la actualidad, solo pueden venir verificadas por una argumentación moral de carácter universal que garantizaría una explicación post-metafísica a la pregunta aludida con anterioridad: ¿por qué aceptamos las normas morales? Es quizá en este punto donde la argumentación que la dialógica y Habermas tienen en mente puede llegar a orientarse hacia actos de habla regulativos como la ética del discurso defiende.

\section{CONCLUSIÓN}

M. Weber y F. Nietzsche diagnosticaron un mismo proceso socio-histórico desde dos perspectivas metodológicas diferentes. Tanto el "desencantamiento del mundo" (pérdida de sentido) de Weber así como la sentencia "Dios ha muerto" de Nietzsche -que se remonta a Hegel- (la idea de Dios no es capaz de actuar como fuente del código moral o teleológico de las sociedades del siglo XX y XXI), afectaron por igual a la teoría moral de tal manera en los últimos años que se ha mostrado escéptica de postular la idea de una Ética que sea aceptada por todos. Los desarrollos en ética comparada nos permiten observan este hecho mediante el análisis de un cierto 
pluralismo existente entre morales religiosas y proyectos personales de vida buena, así como la existencia de distintas teorías desde la filosofía moral (eudemonismo, kantismo, utilitarismo, hedonismo, etc.) donde ninguna de ellas es hegemónica, es decir, ninguna es la Ética (Gómez-Heras, 2003: 21-43).

Es en este politeísmo de los valores donde uno de los caminos posibles para una reconstrucción de la teoría moral aceptada por todos hoy quizá sería una filosofía práctica dirigida hacia la universalización de los intereses y la justica; una teoría moral (de mínimos) donde las nociones de ética y moral, aparentemente separadas, presentan procesos de hibridación en la argumentación moral y en el consenso, ya que los sujetos que argumentan tienen preferencias y valores que son analizados en un terreno común mediante la argumentación y la deliberación (Dryzek, 2010: 93 y ss.), como numerosos ejemplos empíricos señalan en su aplicación práctica a varios países (Niemeyer, 2019: 32). No es caso paradójico observar cómo en la búsqueda de fundamentos racionales sobre los que asentar una moralidad secularizada, independiente de los supuestos de la metafísica y la religión, Habermas y la dialógica -sin olvidar a Apel- coinciden a la hora de reconstruir y reformular la praxis humana mediada pragmáticamente por el uso del lenguaje.

Es cierto que la teoría moral kantiana logró hacer del "imperativo categórico" un criterio para la universalización de las normas o máximas éticas (Kant, 2002: 104 y ss., 116 y ss.). ¿Qué debo hacer? era la pregunta clave para Kant a la hora de definir el espacio propio de los problemas prácticos del ser humano (perspectiva monológica), pero hoy día como hemos intentado mostrar a lo largo de las páginas de este artículo y después de Weber, Nietzsche, el giro lingüístico (Rorty) o el giro pragmático (Bernstein), entre otros, esta pregunta se desplazaría en filosofía moral hacia una ética normativa del ipor qué debo? (perspectiva dialógica). Es en este punto donde la ética del discurso y la dialógica vista desde una perspectiva moral suponen una importante transformación de la ética kantiana, puesto que el carácter monológico del "imperativo categórico" es superado y ampliado por el carácter dialógico del procedimiento discursivo de la argumentación moral. Es en estos términos donde "la validez" del deber ser (deontología) no estaría manifestado en el imperativo de una autoridad particular, sino en la de una voluntad general compartida que será determinable discursivamente, inteligible cognitivamente y visible desde la perspectiva de los diferentes participantes en la búsqueda del consenso a través del mejor argumento; un proceso que en clave constructivista estaría muy ligado a la ontogénesis del Homo sapiens en su proceso de hominización y humanización entendido no sólo como acción racional con respecto a fines de sucesos observables en su modalidad instrumental, sino también desde la conciencia práctico moral de una teoría genética de la acción (Piaget y Kohlberg) en su modalidad comunicativa. Aquí destaca la argumentación moral y sus relaciones con la ética del discurso para una posible Ética que medie deontológicamente en la resolución de los distintos problemas morales y políticos 


\section{ANEXO: EJEMPLO BREVE DE DIÁLOGO PARA UNA TESIS CONDICIONAL}

La siguiente tesis o fórmula sería un ejemplo breve de resolución dinámica de un diálogo condicional siguiendo el marco dialógico estándar presentado con anterioridad $^{12}$. El diálogo condicional sería:

$$
(p \wedge q) \rightarrow p
$$

Siguiendo la lógica dialógica, el diálogo condicional puede llegar a ser analizado hasta su resolución final mediante la coacción sin coacciones del mejor argumento en sentido habermasiano o mediante la regla de ganancia (SR3) de la Escuela de Lille: un diálogo argumentativo finaliza cuando no se pueden ampliar en sucesivos movimientos legales ( $X$ gana sí, y sólo si, es el turno de $Y$ pero no tiene ningún argumento posible). Cuando los participantes ( $\mathbf{P}$ y $\mathbf{O}$ ) en el curso de un proceso de argumentación llegan a la convicción de que han agotado todas las posibles objeciones contra "( $p \wedge q) \rightarrow p$ ", no existirían motivos racionales para seguir manteniendo cualquier actitud hipotética frente a la pretensión de validez sostenida respecto a "( $p \wedge q) \rightarrow p^{\prime}$, es decir, no hay necesidad racional para proseguir la argumentación y " $(p \wedge q) \rightarrow p^{\prime \prime}$ puede ser racionalmente aceptado como se muestra en el siguiente game board (Cuadro 2):

\begin{tabular}{|c|c|c|c|c|c|}
\hline$a$ & $B$ & $c$ & $c^{\prime}$ & $b^{\prime}$ & $a^{\prime}$ \\
\hline & $\mathbf{O}$ & & & $\mathbf{P}$ & \\
\hline & & & & $(p \wedge q) \rightarrow p$ & 0 \\
\hline 1 & $(p \wedge q)$ & 0 & & $\mathrm{p}$ & 4 \\
\hline 3 & $\mathrm{P}$ & & 1 & & 2 \\
\hline
\end{tabular}

Cuadro 2: Diálogo para la tesis " $(p \wedge q) \rightarrow p$ "

\section{Explicación de los distintos movimientos}

\section{1) Movimiento 0}

$$
I_{1}=\{\tau, P-!-(p \wedge q) \rightarrow p\}
$$

" $I_{l} "$ = se refiere al primer movimiento siguiente la regla de partícula para el condicional $(\rightarrow)$.

" $\tau$ " = significa que esta fórmula es una tesis.

\footnotetext{
12 El siguiente ejemplo adaptado procede de la obra de dos discípulos de Shahid Rahman (Redmond y Fontaine, 2011: 28-30). En la obra se presentan multitud de ejemplos para la lógica clásica, intuicionista, lógica de primer orden, lógica modal, etc.
} 
"P $-!-(p \wedge q) \rightarrow p^{\prime \prime}=$ El proponente $(\mathbf{P})$ afirmó un condicional en el proceso de la argumentación y debe ser defendido.

A partir de aquí se inicia una jugada condicional, esto es, un conjunto de movimientos que conciernen a la partícula " $\rightarrow$ ".

2) Movimiento 1

$$
I_{2}=\{\mathrm{CH}, \mathrm{O}-!-(\mathrm{p} \wedge \mathrm{q})\}\left(=C_{1}\right)
$$

El oponente $(\mathrm{O})$ ataca el Movimiento $O$ demandando una justificación para " $\mathrm{p}$ " reconociéndolo en la parte izquierda del condicional. El reconocimiento es una conjunción $(\wedge)$.

Aquí comienza un nuevo juego equivalente a $C_{1}$ para $\mathrm{X}=\mathrm{O}$. Esto significa que atacando el condicional $\mathrm{O}$ está jugando una conjunción introducida por vez primera en el diálogo dando lugar a un nuevo conjunto de movimientos que seguirán las reglas de partículas (en este caso las reglas de conjunción). Esta es una jugada de conjunción: un conjunto de movimientos acerca de la partícula " $\wedge$ ".

" $C_{1}$ " = significa que se ha realizado el primer movimiento siguiendo las reglas de partículas para la conjunción $(\wedge)$.

3) Movimiento 2

$$
I_{3}=\left\{\mathbf{C H}, \mathbf{P}-\langle\text { ataque } 2>\}=\left\{\mathbf{C H}, \mathbf{P} \mathbf{O}-?-\wedge_{1}\right\}\left(=C_{2}\right)\right.
$$

$\mathbf{P}$ no puede defenderse respondiendo directamente " $\mathrm{p}$ " porque es una fórmula atómica que aún no ha sido afirmada por $\mathrm{O}$. Solamente $\mathrm{O}$ tendría el derecho de afirmar una fórmula atómica por primera vez (SR3).

El < ataque $2>$ consistiría en responder preguntando por el primer miembro de la conjunción $\left(\wedge_{1}\right)$ : exactamente lo que $\mathbf{P}$ necesita para una defensa del ataque del movimiento 1: " $\mathrm{p}$ ".

$\mathrm{El}<$ ataque $2>$ corresponde a $C_{2}$.

4) Movimiento 3

$$
C_{3}=\{\mathrm{D}, \mathrm{O}-!-p\}
$$

O se defiende del ataque afirmando " $\mathrm{p}$ ". 
En este punto el conjunto de movimientos que conciernen a la partícula " $\square$ " ha finalizado. La jugada de conjunción se considera acabada para este diálogo.

\section{5) Movimiento 4}

$$
I_{3}=\{\mathrm{D}, \mathrm{P}-!-p\}
$$

$\mathbf{P}$ se defiende del ataque del Movimiento 1 con la fórmula atómica " $\mathrm{p}$ " reconocida por $\mathbf{O}$ en el Movimiento 3.

En este punto el conjunto de movimientos que conciernen a la partícula " $\rightarrow$ " ha finalizado. La jugada de condicional se considera acabada para este diálogo.

Score ( () )

El argumento de $\mathbf{P}$ tendría éxito y ganaría con el Movimiento 3: el diálogo ha concluido y está cerrado (para ello los teóricos de la dialógica utilizan el siguiente símbolo: $\odot)$ ). O no puede realizar otros movimientos puesto que la misma fórmula atómica aparece en los dos últimos movimientos: Movimiento 3 y Movimiento 4.

Las posibles objeciones contra " $\mathrm{p}$ " se han agotado. La resolución de la tesis " $p \wedge q) \rightarrow p$ " se ha resuelto y no existen motivos racionales para seguir insistiendo en la validez de la tesis defendida en el diálogo.

*Observación: Es interesante ver que las distintas series (jugadas de condicional, jugadas de conjunción...) pueden ser combinadas por los jugadores para lograr (o no) sus objetivos en el diálogo: la validez del mejor argumento y la aceptabilidad racional (o el consenso).

\section{Resultado}

Tras la resolución argumentativa $\mathbf{O}$ y $\mathbf{P}$ realizan el movimiento, esto es, la oración realizativa en el sentido clásico de Austin u "oración performativa" (performative sentence) (Austin, 1962: 6; Redmond y Fontaine, 2011: 30). 
BiBLIOGRAFíA

ADDISON, John W, HENKIN, Leon y TARSKI, Alfred. (1965). The Theory of Models. Amsterdam: North-Holland.

ANDERSON, Perry. (2013). Tras las huellas del materialismo histórico. Madrid: Editorial Siglo XXI.

APEL, Karl-Otto. (1973). Transformation der Philosophie: Band II. Das Apriori der Kommunikationsgemeinschaft. Frankfurt: Suhrkamp.

AUSTIN, John L. (1962). How to do things with words. Oxford: Oxford University Press.

CORTINA, Adela. (2010). Ética mínima. Introducción a la filosofía práctica. Madrid: Tecnos.

DÍEZ, José A. y ULISES MOULINES, Carlos. (2016). Fundamentos de filosofía de la ciencia. Barcelona: Editorial Ariel.

DRYZEK John S. (1995). "Political and ecological communication". Environmental Politics, 4, pp. 13-30.

. (2000). Deliberative democracy and beyond: liberals, critics and contestations. Oxford: Oxford University Press.

. (2010). Foundations and frontiers of deliberative democracy.

Oxford: Oxford University Press.

FOUCAULT, Michel. (2014). El orden del discurso. Barcelona: Tusquets Editores.

GARCÍA MARZÁ, V. Domingo. (1992). Ética de la justicia. Madrid: Tecnos.

GÓMEZ-HERAS, José María. (2003). Teorías de la moralidad. Introducción a la ética comparada. Madrid: Editorial Síntesis.

HABERMAS, Jürgen. (1981). Theorie des kommunikativen Handelns. Band 1: Handlungsrationalität und gesellschaftliche Rationalisierung. Frankfurt: Suhrkamp.

Frankfurt: Suhrkamp.

(1983a). Moralbewußtsein und kommunikatives Handeln.

. (1983b). La reconstrucción del materialismo histórico. Madrid:

Taurus.

. (2000). Aclaraciones a la ética del discurso. Madrid: Editorial

Trotta. 
. (2008). Pensamiento postmetafísico. México: Taurus.

. (2011a). Teoría de la acción comunicativa: complementos y estudios previos. Madrid: Cátedra. . (2011b). Verdad y justificación. Madrid: Editorial Trotta.

HINTIKKA, Jaako y SANDU, Gabriel. (2006). "What is logic?", en Jacquette, Dale (ed.), Philosophy of Logic (Handbook of the Philosophy of Science). Amsterdam: Elsevier, pp. 13-39.

HODGES, Wilfrid. (1986). "Truth in a structure". Proceedings of the Aristotelian Society, 86, pp. 135-151.

KANT, Immanuel. (2002). Fundamentación para una metafísica de las costumbres. Madrid: Alianza Editorial.

LORENZEN, Paul. (1955). Einführung in die operative Logik und Mathematik. Berlin: Springer.

Institut. . (1969). Normative logic and ethics. Manheim: Bibliographisches

LORENZEN, Paul y LORENZ, Kuno. (1978). Dialogische Logik. Darmstadt: Wissenschaftstheorie Buchgesellschaft.

MANZANO, María. (1989). Teoría de Modelos. Madrid: Alianza Editorial.

MARTIN-LÖF, Per. (1996). "On the meanings of the logical constants and the justifications of the logical laws". Nordic Journal of Philosophical Logic, 1, pp. 11-60.

MOSTERÍN, Jesús. (2000). Los lógicos. Madrid: Espasa Calpe.

NIEMEYER, Simon. (2019). "Intersubjetive Reasoning in Political Deliberation. A Theory and Method for Assessing Deliberative Transformation at Small and Large Scale". Consultado el 22 de diciembre de 2019. Centre for Deliberative Democracy and Global Governance, en https:/www.governanceinstitute.edu.au/magma/media/upload/ckeditor/files/Intersubj ective\%20Reasoning(4).pdf

RAHMAN, Shahid y KEIFF, Laurent. (2005). "On how to be a dialogician? A short overview on recent developments on dialogues and games", en Vanderveken, Daniel (ed.), Logic, thought and action. Dordrecht: Springer, pp. 359-408. 
RAHMAN, Shahid, STREET, Tony y TAHIRI, Hassan. (2008). The unity of science in the arabic tradition: science, logic, epistemology and their interactions. Dordrecht: Springer.

RAHMAN, Shahid y REDMOND, Juan. (2016). "Armonía dialógica: tonk, Teoría constructiva de Tipos y reglas para jugadores anónimos". Theoria, 31, pp. 27-53.

REDMOND, Juan y FONTAINE, Matthieu. (2011). How to play dialogues. An introduction to dialogical logic. London: College Publications.

REDMOND, Juan y LÓPEZ-ORELLANA, Rodrigo. (2018). "Lógica clásica y esquizofrenia: por una semántica lúdica". Revista de Filosofía, 74, pp. 215-241.

ROMERO, Javier. (2020). "Biosemiótica. Hacia una teoría general de los signos de la naturaleza humana y no humana", Signa. Revista de la Asociación Española de Semiótica, no 29, (en imprenta).

ROMERO, Javier y MEJÍA FERNÁNDEZ, Ricardo. (2019). “La teoría antropológica de Jürgen Habermas: un naturalismo débil entre Kant y Darwin", Cuadernos salmantinos de filosofía, 46, pp. 113-140.

SEARLE, John. (1969). Speech acts. An essay in the philosophy of language. Cambridge: Cambridge University Press.

ULISES MOULINES, Carlos. (1996). "Las ideas básicas del estructuralismo metacientífico". Revista de Filosofía, 16, pp. 93-104.

VAN BENTHEM, Johan. (1991). "General dynamics". Theoretical Linguistic, 17, pp. 159-201.

. (2006a). "Adiós a la soledad: modas dinámicas en la lógica actual". Azafea. Revista de filosofía, 8, pp. 21-33.

pp. 117-122.

. (2006b). "Where is logic going, and should it?". Topoi, 25,

. (2011). Logical dynamics of information and interaction.

Cambridge: Cambridge University Press.

. (2014). Logic in games. Cambridge MA: The MIT Press.

WITTGENSTEIN, Ludwig. (1953). Philosophical Investigations. Oxford: Basil Blackwell.

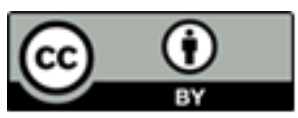

